PROCEEDINGS OF THE AMERICAN MATHEMATICAL SOCIETY

Volume 124, Number 10, October 1996

\title{
ON A CONJECTURE OF S. REICH
}

\author{
PETER Z. DAFFER, HIDEAKI KANEKO, AND WU LI
}

(Communicated by Dale Alspach)

\begin{abstract}
Simeon Reich (1974) proved that the fixed point theorem for single-valued mappings proved by Boyd and Wong can be generalized to multivalued mappings which map points into compact sets. He then asked (1983) whether his theorem can be extended to multivalued mappings whose range consists of bounded closed sets. In this note, we provide an affirmative answer for a certain subclass of Boyd-Wong contractive mappings.
\end{abstract}

\section{INTRODUCTION}

Let $(X, d)$ be a complete metric space. Denote by $C B(X)$ and $K(X)$ the space of all nonempty closed and bounded subsets of $X$ and the space of all nonempty compact subsets of $X$, respectively. The Hausdorff metric induced by $d$ will be denoted by $H$. In [5, p. 40], Reich proved that a mapping $T: X \rightarrow K(X)$ has a fixed point in $X$ if it satisfies $H(T x, T y) \leq k(d(x, y)) d(x, y)$ for all $x, y \in X$ with $x \neq y$, where $k:(0, \infty) \rightarrow[0,1)$ satisfies $\lim \sup _{r \rightarrow t^{+}} k(r)<1$ for every $t \in(0, \infty)$. This result generalizes the fixed point theorem for single-valued mappings that was proved by Boyd and Wong [1]. One of the conjectures made by Reich in [6, 7] asks whether or not the range of $T$ can be relaxed. Specifically the question is whether or not the range of $T, K(X)$, can be replaced by $C B(X)$. In response to Reich's conjecture, the following theorem was recently proved by Mizoguchi and Takahashi [4], and other proofs have been given by Daffer and Kaneko [3] and Tong-Huei Chang [2].

Theorem 1. Let $(X, d)$ be a complete metric space and $T: X \rightarrow C B(X)$. Assume that $T$ satisfies

$$
H(T x, T y) \leq k(d(x, y)) d(x, y)
$$

for all $x, y \in X$ with $x \neq y$, where $k:(0, \infty) \rightarrow[0,1)$ satisfies $\lim \sup _{r \rightarrow t^{+}} k(r)<1$ for every $t \in[0, \infty)$. Then $T$ has a fixed point in $X$.

The stronger condition assumed on $k$ in Theorem 1, viz., $\lim \sup _{r \rightarrow t^{+}} k(r)<1$ for every $t \in[0, \infty)$, implies that $k(t)<h$ for some $0<h<1$ and for small $t>0$. Therefore, with this condition, one may conclude that a mapping satisfying (1) is a contraction in the sense of Banach over a region for which $d(x, y)$ is sufficiently small. In this paper, we obtain a theorem which replaces the closed interval $[0, \infty)$ of this result with the open interval $(0, \infty)$ of the classical Boyd-Wong fixed point theorem.

Received by the editors April 5, 1995.

1991 Mathematics Subject Classification. Primary 47H10.

(C)1996 American Mathematical Society 


\section{The MAIN RESUlT}

In recent papers $([2,8])$ the following class of functions was introduced and studied.

Definition 2. Let $\phi: R_{+} \rightarrow R_{+}$. The function $\phi$ is said to satisfy the condition $(\Phi)$ (denoted $\phi \in(\Phi)$ ) if (i) $\phi(t)<t$ for all $t \in(0, \infty)$; (ii) $\phi$ is upper semicontinuous from the right on $(0, \infty)$; and (iii) there exists a positive real number $s$ such that $\phi$ is nondecreasing on $(0, s]$ and $\sum_{n=0}^{\infty} \phi^{n}(t)<\infty$ for all $t \in(0, s]$.

Chang [2] observed that if $k:(0, \infty) \rightarrow[0,1)$ satisfies $\lim \sup _{r \rightarrow t^{+}} k(r)<1$ for every $t \in[0, \infty)$, then there exists a function $\phi \in(\Phi)$ such that $k(t) t \leq \phi(t)$ for all $t \in(0, \infty)$.

Subsequently, Chang proved the following theorem that generalizes Theorem 1 above.

Theorem 3. Let $(X, d)$ be a complete metric space. Let $T: X \rightarrow C B(X)$ and suppose that there exists a function $\phi \in(\Phi)$ such that

$$
H(T x, T y) \leq \phi\left(\max \left\{d(x, y), d(x, T x), d(y, T y), \frac{d(x, T y)+d(y, T x)}{2}\right\}\right)
$$

for all $x, y \in X$. Then $T$ has a fixed point in $X$.

The purpose of this note is to establish a class of functions that satisfy the inequality $\lim \sup _{r \rightarrow t^{+}} k(r)<1$ for every $t \in(0, \infty)$ and that belong to $(\Phi)$. Utilizing the result of Chang above, we can then obtain a fixed point theorem for multivalued functions that satisfy the conditions required in the conjecture of Reich. The existence of such a class of functions confirms that the conjecture of Reich is still open and that a further investigation toward a complete resolution is required.

Lemma 4. Suppose $\varphi(t)=t-a t^{b}$, where $a>0$.

(i) If $b \geq 2$, then $\sum_{n=0}^{\infty} \varphi^{n}(t)=+\infty$, for all real numbers $t$ except for $t=0$ and $t=a^{-\frac{1}{b-1}}$

(ii) If $1<b<2$, then the series $\sum_{n=0}^{\infty} \varphi^{n}(t)$ converges if $0 \leq t \leq a^{-\frac{1}{b-1}}$ and it diverges if $t<0$ or $t>a^{-\frac{1}{b-1}}$, where $\varphi^{n}=\varphi \circ \cdots \circ \varphi$ denotes the $n$-fold composition.

Proof. A sketch shows that $\sum_{n=0}^{\infty} \varphi^{n}(t)=+\infty$ for $t<0$ or $a^{-\frac{1}{b-1}}<t$, for any value of $b$ greater than 1 . Also, it is clear that the series converges for $t=0$ and $t=a^{-\frac{1}{b-1}}$. Thus we restrict our attention to the case when $t \in\left(0, a^{-\frac{1}{b-1}}\right)$.

For $t \in\left(0, a^{-\frac{1}{b-1}}\right), 0<\varphi(t)<t$. Since $\left\{\varphi^{n}(t): n=1,2, \ldots\right\}$ is a strictly monotone decreasing sequence and bounded below by 0 , it has a limit $z$. Since $\varphi$ is a continuous function, we have $\varphi(z)=\lim _{n \rightarrow \infty} \varphi\left(\varphi^{n}(t)\right)=z$. As a consequence, $z=0$. That is,

$$
\lim _{n \rightarrow \infty} \varphi^{n}(t)=0 \quad \text { for } 0<t<a^{-\frac{1}{b-1}} .
$$

Let $b \geq 2$ and choose an integer $k>a$. Then $\frac{1}{n}-\frac{a}{n^{b}} \geq \frac{1}{n}-\frac{a}{n^{2}} \geq \frac{1}{n+k}$, for all $n \geq \frac{a k}{k-a}=: m_{0}$. Thus $\varphi\left(\frac{1}{n}\right) \geq \frac{1}{n+k}$ for $n \geq m_{0}$. On the interval $\left(0,(a b)^{-\frac{1}{b-1}}\right), \varphi$ is increasing. Let $\epsilon=\min \left\{\frac{1}{m_{0}},(a b)^{-\frac{1}{b-1}}\right\}$. Then

$$
\varphi(t) \geq \varphi\left(\frac{1}{m_{t}}\right) \geq \frac{1}{m_{t}+k} \quad \text { for } 0<t<\epsilon,
$$


where $m_{t}:=\left[\frac{1}{t}+1\right] .([x]$ denotes the largest integer less than or equal to $x$.

Now let $t \in\left(0, a^{-\frac{1}{b-1}}\right)$. By (2), there exists a positive integer $n_{0}$ such that $0<\varphi^{n}(t)<\epsilon$ for $n \geq n_{0}$. Let $r:=\frac{1}{\varphi^{n}(t)}$. Then it follows that

$$
\varphi^{n}(t) \geq \frac{1}{r+k\left(n-n_{0}\right)} \quad \text { for } n \geq n_{0} .
$$

Indeed, this is true for $n=n_{0}$, and we see that, for $n \geq n_{0}$,

$$
\begin{aligned}
\varphi^{n+1}(t) & =\varphi\left(\varphi^{n}(t)\right) \\
& \geq \varphi\left(\frac{1}{r+k\left(n-n_{0}\right)}\right) \geq \frac{1}{r+k\left(n-n_{0}\right)+k}=\frac{1}{r+k\left(n+1-n_{0}\right)},
\end{aligned}
$$

where we use the induction assumption for the first inequality. The induction method proves (4). Hence,

$$
\sum_{n=1}^{\infty} \varphi^{n}(t) \geq \sum_{n=n_{0}}^{\infty} \varphi^{n}(t) \geq \sum_{n=n_{0}}^{\infty} \frac{1}{r+k\left(n-n_{0}\right)}=+\infty .
$$

Now let $1<b<2$. For $1<c<\frac{1}{b-1}$, we have

$$
n^{b c}\left(\frac{1}{n^{c}}-\frac{1}{(n+1)^{c}}\right)=\frac{n^{b c}\left((n+1)^{c}-n^{c}\right)}{n^{c}(n+1)^{c}}=\frac{n^{b c-c-1}}{\left(1+\frac{1}{n}\right)^{c}} \cdot \frac{\left(1+\frac{1}{n}\right)^{c}-1}{\frac{1}{n}} \rightarrow 0
$$

as $n \rightarrow \infty$. Thus, we can choose $n_{0}$ such that $n_{0}^{-c}<(a b)^{-\frac{1}{b-1}}$ (so that $\frac{1}{n_{0}^{c}}$ is in the interval where $\varphi$ is increasing) and $\frac{1}{n^{c}}-\frac{1}{(n+1)^{c}} \leq \frac{a}{n^{b c}}$, for all $n \geq n_{0}$. As a result, we have $\varphi\left(\frac{1}{n^{c}}\right) \leq \frac{1}{(n+1)^{c}}$ for all $n \geq n_{0}$. For every $t \in\left[0, a^{-\frac{1}{b-1}}\right], \lim _{n \rightarrow \infty} \varphi^{n}(t)=0$ (cf. (2)), and so for some integer $k_{0}$ we have $\varphi^{k_{0}}(t) \leq \frac{1}{n_{0}^{c}}$.

We claim that $\varphi^{n}(t) \leq \frac{1}{\left(n_{0}+n-k_{0}\right)^{c}}$, for all $n \geq k_{0}$. Indeed, this is true for $n=k_{0}$, and we have that

$$
\varphi^{n+1}(t)=\varphi\left(\varphi^{n}(t)\right) \leq \varphi\left(\frac{1}{\left(n_{0}+n-k_{0}+1\right)^{c}}\right) \leq \frac{1}{\left(n_{0}+n+1-k_{0}\right)^{c}},
$$

where the induction assumption is used for the first inequality. This completes the proof of our claim. Hence

$$
\sum_{n=0}^{\infty} \varphi^{n}(t)=\sum_{n=0}^{k_{0}-1} \varphi^{n}(t)+\sum_{n=k_{0}}^{\infty} \varphi^{n}(t)=\sum_{n=0}^{k_{0}-1} \varphi^{n}(t)+\sum_{n=k_{0}}^{\infty} \frac{1}{\left(n_{0}+n-k_{0}\right)^{c}}<+\infty
$$

since $c>1$.

Theorem 5. Let $(X, d)$ be a complete metric space and $T: X \rightarrow C B(X)$. If there exists an upper right semi-continuous function $\varphi: R_{+} \rightarrow R_{+}$such that (i) $\varphi(t)<t$ for all $t>0$, (ii) $\varphi(t) \leq t-a t^{b}, a>0$, for some $1<b<2$ on some interval [0,s], $s>0$, and (iii)

$$
H(T x, T y) \leq \varphi(d(x, y))
$$

for all $x, y \in X$, then $T$ has a fixed point in $X$.

Proof. Writing $\psi(t)=t-a t^{b}$ and $\epsilon=\min \left\{s,(a b)^{\frac{1}{b-1}}\right\}$, we have that $\varphi^{n}(t) \leq \psi^{n}(t)$ for every positive integer $n$ and $t \in[0, \epsilon]$ (an interval upon which $\psi$ is increasing). Indeed, this holds for $n=1$ and if it holds for $n$, then

$$
\psi^{n+1}(t)=\psi\left(\psi^{n}(t)\right) \geq \psi\left(\varphi^{n}(t)\right) \geq \varphi\left(\varphi^{n}(t)\right)=\varphi^{n+1}(t),
$$


since $\psi^{n}(t), \varphi^{n}(t) \in[0, \epsilon]$ for every $n$. This completes the induction proof of $\varphi^{n}(t) \leq$ $\psi^{n}(t)$. By Lemma $4, \psi \in(\Phi)$; hence we also have $\varphi \in(\Phi)$ and the conclusion follows from Theorem 3.

We remark that Theorem 5 can be formulated using the contractive condition

$$
H(T x, T y) \leq \varphi\left(\max \left\{d(x, y), d(x, T x), d(y, T y), \frac{d(x, T y)+d(y, T x)}{2}\right\}\right),
$$

because of Theorem 3 above. However, because of a more relevance to the conjecture of Reich, we chose to describe our theorem using the contractive condition $H(T x, T y) \leq \varphi(d(x, y))$.

Theorem 5 reveals that with $k(t)=1-a t^{b-1}$ we have a class of functions that satisfy all the conditions in the conjecture of Reich. Hence we obtain the following:

Corollary 6. Let $(X, d)$ be a complete metric space and $T: X \rightarrow C B(X)$. Assume that $T$ satisfies

$$
H(T x, T y) \leq k(d(x, y)) d(x, y)
$$

for all $x, y \in X$, where $k: R_{+} \rightarrow[0,1]$ with $k(t)<1$ for $t>0$, and $k(t) \leq 1-a t^{b-1}$, $a>0$, for some $b \in(1,2)$ on some interval $[0, s], 0<s<a^{-\frac{1}{b-1}}$. Then $T$ has $a$ fixed point in $X$.

\section{REFERENCES}

1. D. W. Boyd and J. S. W. Wong, On Nonlinear Contractions, Proc. A.M.S. 89(1968), 458-464. MR 39:916

2. T. H. Chang, Common Fixed Point Theorems for Multi-Valued Mappings, Math. Japonica 41 (1995), 311-320. CMP 1995:11

3. P. Z. Daffer and H. Kaneko, Fixed Points of Generalized Contractive Multi-Valued Mappings, J. Math. Anal. Appl. 192 (1995), 655-666. CMP 1995:12

4. N. Mizoguchi and W. Takahashi, Fixed Points Theorems for Multi-Valued Mappings on Complete Metric Spaces, J. Math. Anal. Appl. 141(1989) 177-188. MR 90f:47086

5. S. Reich, Fixed Points of Contractive Functions, Boll. Un. Mat. Ital., (4) 5 (1972), 26-42. MR 46:8206

6. S. Reich, Some Fixed Point Problems, Atti. Accad. Naz. Lincei, 57(1974), 194-198. MR 53:1346

7. S. Reich, Some Problems and Results in Fixed Point Theory, Contemporary Math. A.M.S. 21(1983), 179-187. MR 85e:47082

8. K. Sastry, S. Naidu and J. Prasad, Common Fixed Points for Mutimaps in a Metric Space, Nonlinear Anal. 13(3) (1989) 221-229. MR 90b:54034

Department of Mathematics, Macon College, Macon, Georgia 31297

E-mail address: pdaffer@cennet.mc.peachnet.edu

Department of Mathematics and Statistics, Old Dominion University, Norfolk, VirGINIA 23529-0077

E-mail address: kaneko@math.odu.edu

Department of Mathematics and Statistics, Old Dominion University, Norfolk, VirGINIA 23529-0077

E-mail address: wuli@math.odu.edu 